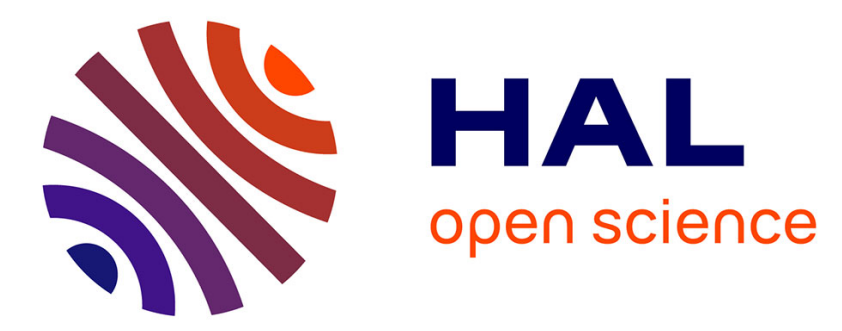

\title{
Imaging and moving a xenon atom on a copper (110) surface with the tip of a scanning tunneling microscope: A theoretical study \\ X. Bouju, Christian Joachim, Christian Girard, P. Sautet
}

\section{- To cite this version:}

X. Bouju, Christian Joachim, Christian Girard, P. Sautet. Imaging and moving a xenon atom on a copper (110) surface with the tip of a scanning tunneling microscope: A theoretical study. Physical Review B: Condensed Matter and Materials Physics (1998-2015), 1993, 47 (12), pp.7454-7461. 10.1103/PhysRevB.47.7454 . hal-01795547

\section{HAL Id: hal-01795547 \\ https://hal.science/hal-01795547}

Submitted on 18 May 2018

HAL is a multi-disciplinary open access archive for the deposit and dissemination of scientific research documents, whether they are published or not. The documents may come from teaching and research institutions in France or abroad, or from public or private research centers.
L'archive ouverte pluridisciplinaire HAL, est destinée au dépôt et à la diffusion de documents scientifiques de niveau recherche, publiés ou non, émanant des établissements d'enseignement et de recherche français ou étrangers, des laboratoires publics ou privés. 


\title{
Imaging and moving a xenon atom on a copper (110) surface with the tip of a scanning tunneling microscope: A theoretical study
}

\author{
X. Bouju \\ Laboratoire de Physique Moléculaire, Université de Besançon, 25030 Besançon CEDEX, France
}

\author{
C. Joachim
}

Groupe d'Electronique Moléculaire, CNRS, 29 Rue J. Marvig, Bô̂te Postale 4347, 31077 Toulouse CEDEX, France

C. Girard

Laboratoire de Physique Moléculaire, Université de Besançon, 25030 Besançon CEDEX, France

P. Sautet

Laboratoire de Chimie Théorique, Ecole Normale Supérieure, 46 allée d'Italie, 69364 Lyon CEDEX 07, France

(Received 2 March 1992; revised manuscript received 4 November 1992)

The sliding of a xenon atom on a $\mathrm{Cu}(110)$ surface caused by a scanning tunneling microscope (STM) tip is discussed. Initially, the stable height and lateral position of a $\mathrm{Xe}$ atom on $\mathrm{Cu}(110)$ in the absence of the tip is calculated by considering Born-Mayer repulsive and van der Waals interactions. Using this geometry, a constant-current STM image of the Xe atom is then calculated using the STM-ESQC technique. For $I=1 \mathrm{nA}$ and $V=10 \mathrm{mV}$, a bump of $1.7 \AA$ is found, which is in good agreement with experiments. The stability of the Xe atom under the tip apex as a function of the tip to substrate distance is also discussed. At each tip height, the tunnel current intensity is calculated. The threshold tunnel resistance required to move a Xe atom with the tip is deduced from these calculations and is in good agreement with the experimental one. It is argued that the sliding process is due to the trapping of the Xe atom in a van der Waals well created by the tip apex. This trap is moved along the ( 110$)$ rows as the tip scans.

\section{INTRODUCTION}

The adsorption of rare-gas atoms on surfaces has been the subject of extensive experimental investigations. ${ }^{1-4}$ It is well known that on metal, standard methods such as low-energy electron diffraction and elastic neutron scattering can be used to determine the average structure of a rare-gas monolayer on a metallic surface. The main drawback of these techniques is the difficulty of obtaining the local arrangement of the adsorbates. With the advent of the scanning tunneling microscope (STM), one can obtain atomic-resolved images of a large variety of adsorbates on metal $^{5}$ and semiconductor ${ }^{6}$ surfaces. Consequently, the STM allows the adsorption sites to be located and the adsorbate-substrate interaction potentials to be determined.

Recently, a series of experiments have demonstrated the capability of the STM to move atoms or molecules adsorbed on metal or semiconductor surfaces. ${ }^{7-13}$ In a pioneering work, Eigler and Schweizer ${ }^{7}$ have used a tungsten tip to position with subnanometer precision individual xenon atoms adsorbed on a single-crystal (110) nickel surface. In similar experimental conditions, the reversible transfer of adsorbed atoms between the $\mathrm{Ni}(110)$ surface and the tungsten tip has been demonstrated using a pulse bias voltage technique. ${ }^{9}$

The adsorption energy of $\mathrm{Xe}$ on $\mathrm{Ni}(110)$ is low $(200$ meV). ${ }^{4}$ Therefore, atom-push manipulations must be performed at low temperature $(4.2 \mathrm{~K})$ and in ultrahigh vacuum. In such conditions, very well-resolved images of $\mathrm{Xe}$ atomic arrays can be recorded by scanning at a tipsample distance sufficiently large for the $\mathrm{Xe}$ atoms to remain in their equilibrium sites. ${ }^{7}$ When the tip-surface distance is reduced, it has been proposed that the forces between the tip apex and the $\mathrm{Xe}$ atom responsible for the Xe sliding along the (110) rows are of the van der Waals type, rather than of the electrostatic type. ${ }^{7}$

A detailed interpretation of these experiments requires quantitative data for the $\mathrm{Xe}-\mathrm{Ni}(110)$ surface-tip-apex interactions. If the surface crystallographic structure is taken into account, the $\mathrm{Xe-Ni(110)}$ distance can be deduced from the computed STM images, and the adsorption sites can be recognized in these images. However, little has been done to predict how a rare-gas atom may look on the surface of a metal when it is imaged with an STM. In a recent contribution, Eigler et $a l .{ }^{8}$ have used the atom-on-jellium model to calculate the apparent height of a single Xe atom in a constant-current STM image. These authors conclude that the $\mathrm{Xe} 6 s$ resonance tail is the major contributor to the Fermi-level local-state density that permits such an adsorbate to be imaged. But the surface crystal structure and the atomic structure of the tip were not considered in the calculation. ${ }^{8}$

In this paper, we present a theoretical study of the stability of Xe atoms placed between a STM tip and the (110) face of a fcc metallic substrate. We also present a calculation of the tunnel current between the probe tip and the adsorbate-substrate system. For this study, we 
have chosen the (110) face of copper, for which extensive experimental results with physisorbed rare-gas atoms are available in the literature. ${ }^{14}$ This standard system displays dynamical and structural characteristics very close to the $\mathrm{Ni}-\mathrm{Xe}$ system studied by Eigler and coworkers.

This paper is organized as follows. In Sec. II, the stability of a Xe atom on $\mathrm{Cu}(110)$ is analyzed using a discrete-potential model. The potential is the sum of long-range dispersion terms and of short-range BornMayer terms characterizing the repulsive effects between the atom and the surface. Our method is similar to that of Hill, Haller, and Celli ${ }^{15}$ and Vidali, Cole, and Klein ${ }^{16}$ since it relies on the knowledge of a reduced number of parameters describing the atom and the surface (atomsurface dispersion coefficient, short-range repulsive parameters, and crystallographic characteristics of the surface). The van der Waals coefficients are related to the dipolar and quadrupolar fluctuations of both the adsorbate and the atoms of the surface. ${ }^{16}$ In Sec. III, we present theoretical STM images of a single Xe atom. The tunneling current between the tip and the substrate through the xenon is calculated from the generalized Landauer formula. ${ }^{17}$ The electronic structure of the complete system (tip, surface, and adsorbate) is obtained by molecular orbitals which are calculated using the extended-Hückel-molecular-orbital (EHMO) method. ${ }^{18}$ In Sec. IV, the physical mechanisms responsible for the sliding of a Xe atom are analyzed by introducing the whole potential experienced by Xe within the gap between the tip and the substrate. Finally, our results are compared with the experimental sliding-threshold tunnel resistance found by Eigler.

\section{THE STABILITY OF A Xe ATOM ON THE Cu(110) SURFACE}

The interaction energy $U$ between a Xe atom and the copper crystal surface can be decomposed into a shortrange Born-Mayer part $U_{S}$ and a long-range dispersionenergy part $U_{D}$ with

$$
U_{S}=\sum_{n_{1}, n_{2}} \sum_{p} A_{0} \exp \left[-\left(\alpha_{\mathrm{Xe}-\mathrm{Cu}}\right)\left|\mathbf{R}-\mathbf{r}_{n_{1}, n_{2}, p}\right|\right]
$$

and

$U_{D}=-\frac{\hbar}{2 \pi} \int_{0}^{+\infty} d \xi \int d \mathbf{r} d \mathbf{r}^{\prime} \chi_{a}\left(\mathbf{r}^{\prime}, \mathbf{r}, i \xi\right) K_{S}\left(\mathbf{r}, \mathbf{r}^{\prime}, i \xi\right)$.

In (1) and (2), the lattice vector $\mathbf{r}_{n_{1}, n_{2}, p}$ is defined by

$$
\mathbf{r}_{n_{1}, n_{2}, p}=\left(n_{1} \mathbf{a}_{1}+n_{2} \mathbf{a}_{2}+\tau_{p}, z_{p}\right)
$$

where $a_{1}$ and $a_{2}$ are the bidimensional primitive translation vectors on the surface, and $n_{1}, n_{2}$ are integer numbers. $\tau_{p}$ defines the relative position of atoms in a given cell and two different planes, and $z_{p}$ defines the coordinate of a plane with respect to the $z=0$ crystal surface. Note that in (1), the vector $\mathbf{R}(1, d)$ gives the location of the Xe atom on the surface, $d$ being the Xe-copper surface distance.

The Born-Mayer part $U_{S}$ of $U$ accounts for the repul- sion between $\mathrm{Xe}$ and the surface copper atoms. The short-range parameter $A_{0}$ is determined for the $\mathrm{Cu}(110)$ face by fitting the total energy $U$ to the experimental value for the adsorption energy at zero coverage. ${ }^{4}$

In contrast with this semiempirical estimation of the short-range energy $U_{S}$, the long-range dispersion energy $U_{D}$ can be determined using the generalized susceptibility theory. ${ }^{19}$ In (2), $\chi_{a}$ is the linear susceptibility of the Xe atom and $K_{S}$ the generalized susceptibility of the electric potential generated by the metal surface. ${ }^{20}$ For a single atom or a small molecule, the multipolar expansion of $\chi_{a}$ is valid

$$
\begin{aligned}
\chi_{a}\left(\mathbf{r}^{\prime}, \mathbf{r}, i \xi\right)= & \alpha_{a}^{1}(i \xi)[2] \nabla_{\mathbf{r}^{\prime}} \nabla_{\mathbf{r}}\left(\mathbf{r}^{\prime}-\mathbf{R}\right) \delta(\mathbf{r}-\mathbf{R}) \\
+ & \frac{1}{9} \alpha_{a}^{(2)}(\xi)[4] \nabla_{\mathbf{r}^{\prime}}^{(2)} \nabla_{r}^{(2)} \\
& \times \delta\left(\mathbf{r}^{\prime}-\mathbf{R}\right) \delta(\mathbf{r}-R)+\cdots,
\end{aligned}
$$

where $\alpha_{a}^{(1)}(\omega)$ and $\alpha_{a}^{(2)}(\omega)$ represent both the dipolar and quadrupolar polarizabilities of the isolated Xe atom. Using (4) to calculate $U_{D}$, it comes from (2)

$$
\begin{aligned}
U_{D}=-\frac{\hbar}{2 \pi} \int_{0}^{+\infty} d \xi & \left\{\mathbf{S}_{S}^{(1)}(\mathbf{R}, \mathbf{R}, i \xi)[2] \alpha_{a}^{(1)}(i \xi)\right. \\
& \left.+\frac{1}{9} \mathbf{S}_{S}^{(1)}(\mathbf{R}, \mathbf{R} i \xi)[4] \alpha_{a}^{(2)}(i \xi)\right\} .
\end{aligned}
$$

In (4) and (5), the conventional notation $[k]$ is used for a tensor contraction of $k$ th order. The tensors $\mathbf{S}^{(k)}$ are defined by

$$
\mathbf{S}^{(k)}(\mathbf{R}, \mathbf{R}, i \xi)=\nabla_{\bar{r}}^{(k)} \nabla_{\bar{r}^{\prime}}^{(k)} K_{S}\left(\mathbf{r}, \mathbf{r}^{\prime}, i \xi\right) .
$$

After further calculations, $U_{D}$ given by (5) can be separated into a continuum part $\bar{U}_{D}$ and a corrugation part $\widetilde{U}_{D}$ with

$$
\bar{U}_{D}(\mathbf{R})=-b \sum_{p=0}^{\infty}\left\{\frac{3 C_{3}}{(d+p b)^{4}}+\frac{5 C_{5}}{(d+p b)^{6}}\right\}
$$

and

$$
\begin{aligned}
\widetilde{U}_{D}(\mathbf{R})=- & b \sum_{p=0}^{\infty} \sum_{g} \operatorname{cosg} \cdot\left(1+\tau_{p}\right) \\
\times & {\left[\frac{3 C_{3} g^{2}}{(d+p b)^{2}} K_{2}[g(d+p b)]\right.} \\
& \left.+\frac{5 C_{5} g^{3}}{(d+p b)^{3}} K_{3}[g(d+p b)]\right],
\end{aligned}
$$

where $b=\left|Z_{p+1}-Z_{p}\right|$ is the distance between two successive planes of the substrate. The van der Waals parameters $C_{3}$ and $C_{5}$ are related to dipole and quadrupole fluctuations. ${ }^{21,22}$ The sum over the reciprocal lattice vector $\mathrm{g}$ runs over the doublet values $\left(g_{1}, g_{2}\right) \neq(0,0)$ which are consistent with the symmetry properties of the lattice. $K_{n}$ is a modified Bessel function of integer order.

From (7) and (8), we calculate the potential energy $U(d)$ of a Xe atoms on the unreconstructed $\mathrm{Cu}(110)$ surface as a function of its distance $d$ to the first copper layer 
of the (110) face (Fig. 1). Four different adsorption sites can be distinguished as indicated in Fig. 2. Their equilibrium distances and holding energies can easily be deduced from the corresponding $U(d)$ curves. The van der Waals parameters $C_{3}$ and $C_{5}$ have been calculated from data taken in Ref. 22. The short-range constant $A_{0}$ in (1) has been fitted in the manner described above and is equal to $7.9 \times 10^{3} \mathrm{eV}$. This leads to a Xe holding energy above the hollow site equal to ${ }^{4} 178 \mathrm{meV}$. In this position, which is the most stable one, the equilibrium distance $d_{\text {eq }}=2.78 \AA$, calculated using the present method, is slightly larger than the one evaluated from a hard-sphere model. The activation energy $E_{D}$ (cf. Fig. 3) required for a Xe atom to jump from one hollow site to another one is found to be lower when the atom moves along the (110) rows $\left(E_{D}=16.5 \mathrm{meV}\right)$ than when it moves perpendicularly to these rows $\left(E_{D}=51 \mathrm{meV}\right)$. This confirms the observation that the hollow sites are the most stable sites for $\mathrm{Xe}$ on the $\mathrm{Cu}(110)$ surface. Top sites are not equilibrium positions for $\mathrm{Xe}$, and they block the lateral diffusion of $\mathrm{Xe}$ at low temperature. These top rows, therefore, form a natural mask on the (110) face. This is very helpful for manipulating atoms.

\section{IMAGING Xe ATOMS ON Cu(110)}

A way of testing our estimated equilibrium position of $\mathrm{Xe}$ on $\mathrm{Cu}(110)$ is to calculate a constant-current STM image of $\mathrm{Xe}$ on $\mathrm{Cu}(110)$. Then, by comparing the experimental and calculated images, we can deduce, for example, the altitude of $\mathrm{Xe}$ on $\mathrm{Cu}(110)$ at low temperature. An explanation of the imaging process for $\mathrm{Xe}$ was proposed recently by Lang and co-workers for $\mathrm{Xe}$ on $\mathrm{Ni}$ (110). It is the tail of the virtual $6 s$-state resonance which provides a nonnegligible tunneling probability at the Fermi energy of the chosen metal. Note that it is now a well-recognized phenomenon that through-bond tunneling occurs via the resonance tail of either filled level or from an empty one. These levels are not in resonance with the bulk Fermi level.

In the following, the STM scans and STM images were

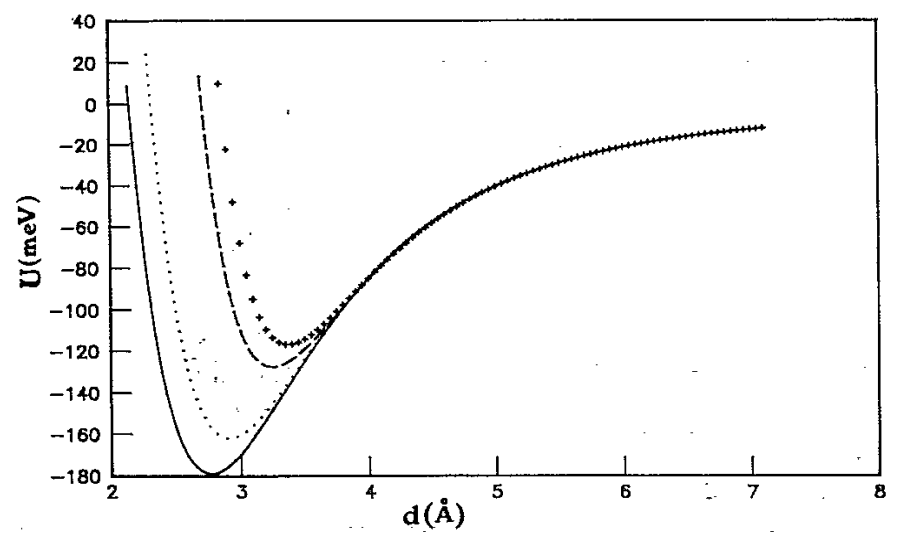

FIG. 1. Holding interaction potential $E(d)$ as a function of the Xe atom-surface distance $d$ for the different adsorption sites defined in Fig. 2: ( $-(-)$ for site $a,(\cdots)$ for site $b,(---)$ for site $c$, and $(++++)$ for site $d$.

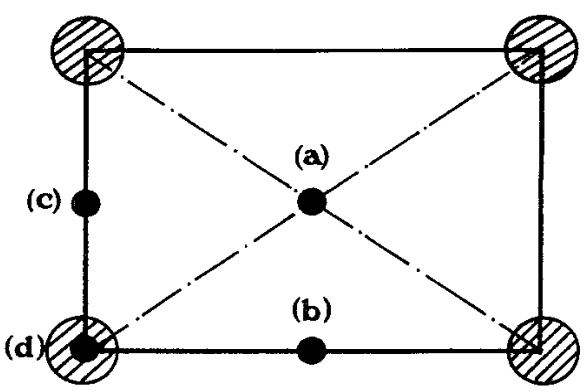

FIG. 2. The four adsorption sites studied on the $\mathrm{Cu}(110)$ surface. Large circles with oblique lines are $\mathrm{Cu}$ atoms and the small black ones are the adsorption sites $(a),(b),(c)$, and $(d)$.

obtained using an extension of our elastic-scatteringquantum-chemistry (ESQC) technique ${ }^{23}$ of calculating images. This extension has already been described in recent contributions. ${ }^{23,24}$ Therefore, we will focus here more on the interpretation of the images rather than on the details of the theory. Note that this STM-EQSC technique has already been used to calculate STM images of benzene on $\mathrm{Rh}(111),{ }^{24} \mathrm{Cu}-\mathrm{Phth}$ alocyanine on $\mathrm{Cu}(100),{ }^{25}$ graphite, ${ }^{26}$ and sulfur on $\operatorname{Re}(0001){ }^{27}$

Let us briefly recall the principal features of our model, which uses the generalized Landauer formula (instead of the Bardeen approximation) to evaluate the tunnelcurrent intensity. When tunneling takes place, the tip is very close (5-7 $\AA$ ) to the substrate. The system to consider can therefore not be the free adsorbate alone. It must be a supermolecule made of the tip apex, the adsorbate, and the substrate surface. ${ }^{24,28}$ The electronic structure of this supermolecule is dependent upon the $(x, y, z)$ coordinates of the STM tip apex relative to the substrate. This supermolecule strongly interacts with the tip wire and with the bulk of the substrate. In this case, for an electron coming from the tip wire (or from the bulk of the substrate), the supermolecule represents a defect compared to the ordered electronic structure met by the elec-

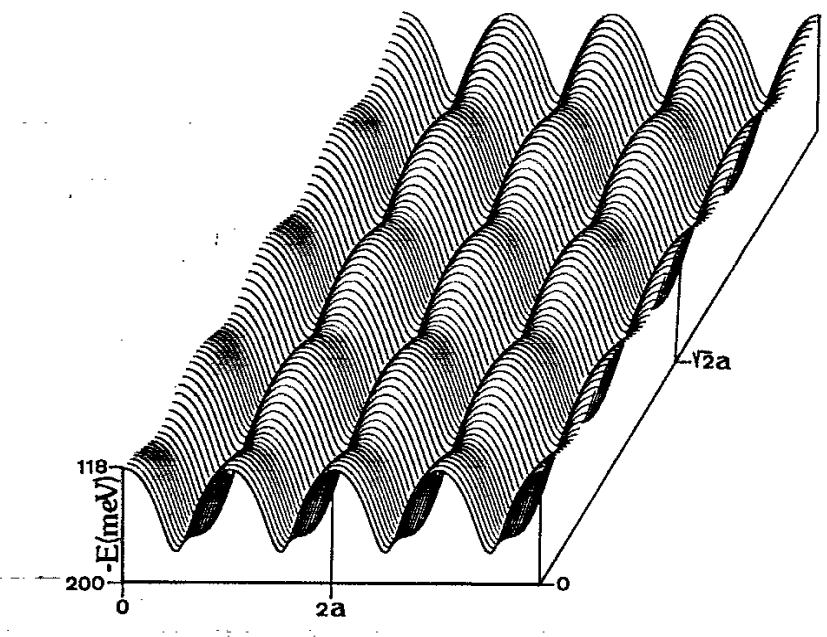

FIG. 3. Map of the minima $E_{\text {bs }}$ of the Xe potential energy $U(d)$ above the (110) $\mathrm{Cu}$ surface. The Xe scanned area is $(4 a \times 2 a \sqrt{2})$ with $a$ the $\mathrm{Cu}$ lattice parameter. The corrugation in the bottom of the ( 110$) \mathrm{Cu}$ rows is $16.5 \mathrm{meV}$. 
trons in the tip and in the substrate. Scattering from this defect is mainly elastic due to the small size of the STM tunneling junction. At low bias voltage $V$, the tunnelcurrent intensity $I(x, y, z)$ through such a $(x, y, z)$ dependent defect is given by the generalized Landauer formula. ${ }^{17}$ This formula relates the conductance $I(x, y, z)$ $V^{-1}$ of the defect to the trace of the squared transmission matrix through the defect taken at the bulk Fermi level. ${ }^{29}$ This matrix is obtained following our ESQC procedure ${ }^{30}$ and using EHMO calculations. ${ }^{18}$ In this manner, we calculate the tunneling-current intensity with the tip apex and surface connected to electron reservoirs without making use of the standard Fermi golden rule (or Bardeen) approximation. ${ }^{31}$ This tunnel current is a function of the Fermi level position relative to the energy of the molecular orbitals of the defect, ${ }^{24}$ i.e, the STM image calculated with this technique depends on the bias voltage. But there is no polarization effect of these orbitals taken into account due to the electric field.

In order to obtain theoretical images that are valuable for determining the structure of $\mathrm{Xe}$ on $\mathrm{Cu}(110)$ and for discussing the sliding process, we need a good description of the electronic structure of the tip-Xe-Cu(110) tunnel junction. The $\mathrm{Cu}(110)$ surface is described here by two layers of copper atoms, each layer being composed of 54 atoms in a $6 \times 9$ rectangular lattice that respects crystallographic distances. The fcc $\mathrm{Cu}$ bulk lattice supporting the $\mathrm{Cu}(110)$ surface is generated by a periodic repetition of these two layers, which gives the bulk regular fcc copper structure. Lateral cyclic boundary conditions are used. The Xe atom is positioned on this surface on a central hollow site that respects the equilibrium position (position $a$ ) found in Sec. II.

The tip apex is itself attached to an identical $\mathrm{Cu}(110)$ surface. This surface is connected to the bulk of the tip wire, which has the same atomic structure as the bulk of the substrate. As shown in Fig. 4 for a line scan that passes over on the Xe atom, the print of the Xe atom on the scan strongly depends on the tip atomic shape. There is no trace of the Xe atom in an image made with a flat tip [curve (1) of Fig. 4]. In this case, it is the Xe atom that plays the role of a tip apex and scans the tip surface.

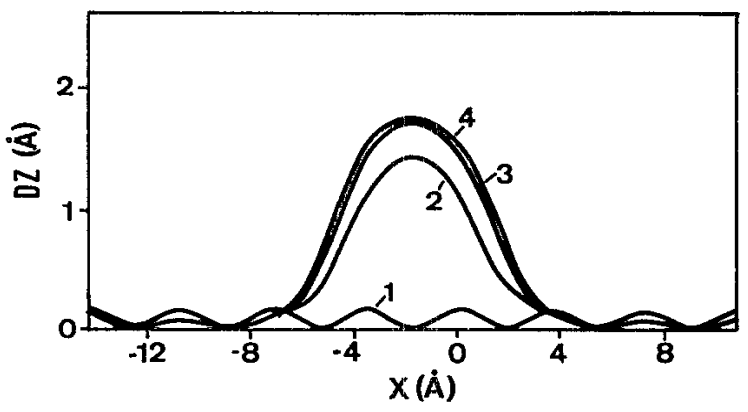

FIG. 4. Variations of the Xe-bump apparent shape as a function of the tip apex chosen. The tip was constant-current scanned orthogonally to the $(110)$ rows for $I=1 \mathrm{nA}$ and $V=10$ $\mathrm{mV}$. (1), no tip apex; (2), one-atom tip apex; (3), a two-layer pyramid five-atom tip apex; and (4), a three-layer pyramid 14-atom tip apex.
Away from this extreme case, the print of the Xe atom becomes independent of the tip-shape geometry when the tip apex is represented by at least two atomic layers. This is due to the size of a Xe atom: the interaction between the tip base and the $\mathrm{Xe}$ atom becomes negligible for a two-layer tip.

For the scans in Fig. 4, each copper atom is described by a $4 s$ Slater atomic orbital with an exponent and an energy given by the standard EHMO parametrization. ${ }^{18}$ However, the extension of this type of orbital in space is not sufficient to ensure that the tunneling current varies by one order of magnitude per $\AA .{ }^{24}$ Therefore, a doublezeta $4 s$ orbital is used for the end atom of the tip. This orbital incorporates a diffuse part so that a better description of long-range interactions with the tip and a good current-distance variation are obtained. Parametrization of the $\mathrm{Xe}$ atom is more critical. Since $\mathrm{Xe}$ is a closed-shell atom, electron tunneling may occur by the virtual creation of an electronic hole in its ground-state configuration or by the virtual occupation of its $6 s$ atomic orbital. The $5 p^{6}$ ground-state configuration of Xe has a high ionization energy. Since the energy level of the $5 p^{6}$ configuration is well below the copper Fermi level, a hole creation in this $5 p^{6}$ configuration is not going to play a first-order role in the tunneling process. On the other hand, the $6 s$ orbital is the first electronic excited state $5 p^{5} 6 s^{1}$ of Xe. Therefore, the $6 s$ virtual orbital has to be considered. The EHMO parameters of this $6 s$ orbital are optimized starting from the cesium atom $6 s$ orbital EHMO parameters because Cs has a $5 p^{6} 6 s^{1}$ electronic configuration in its ground state. Experimental evidence has shown that the $\mathrm{Xe} 6 s$ virtual state is close to the vacuum level. Therefore, since cesium has a very low ionization energy, the EHMO coulomb parameter of this $6 s$ orbital has been derived from the cesium $6 s$ one. The superposition of two $6 s$ Slater orbitals, one with a 2.09 exponent and one with a 1.24 exponent, is used for the spatial part of this parametrization. This parametrization can be optimized using the experimental scans as in any semiempirical calculations. ${ }^{18}$ Notice that we have tested a more complete description of the Xe using the $6 p$ and $5 d$ atomic orbitals. No change in the image was apparent. The $5 d$ is too contracted to modify the tunnel current intensity through the $\mathrm{Xe}$ atom. The $6 p$ do not contribute significantly to the image and was neglected.

For a chosen parametrization, one possible way of comparing theory and experiment is to consider the apparent height of the Xe bump in the STM image as a function of the $\mathrm{Xe}-\mathrm{Cu}$ altitude on the surface. As shown in Fig. 5, the height increase is not large: $0.1 \AA$ for a $0.5-\AA$ increase in the $\mathrm{Xe}-\mathrm{Cu}$ altitude variation. But thanks to the progress in STM instrumentation, this difference can be detected. We have, therefore, a way of extracting the $\mathrm{Xe}-\mathrm{Cu}$ altitude by comparing experimental and theoretical STM images. However, this determination depends critically on the tip-apex structure. Therefore, an effective tip shape must be constructed by considering a scan over a naked surface before the Xe altitude is studied.

Figure 6 presents a three-dimensional image of a calculated constant-current STM image of a single Xe atom 


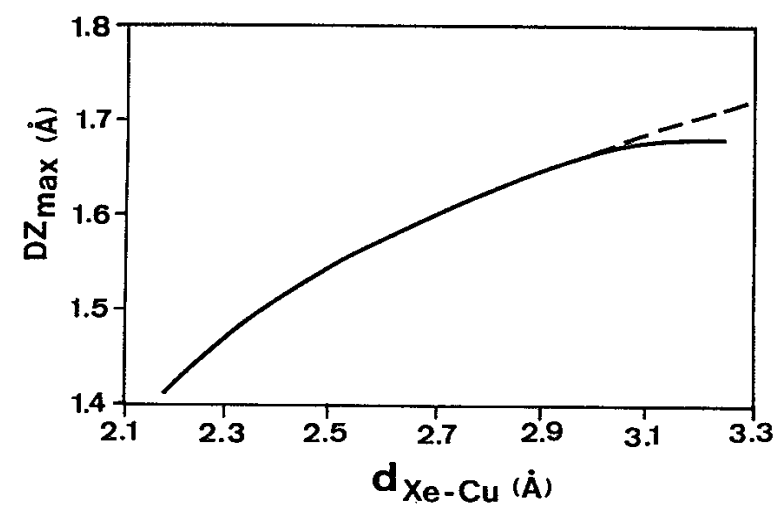

FIG. 5. Variation of the apparent height $D Z_{\max }$ of the $\mathrm{Xe}$ bump in the STM image as a function of the Xe-to-surface distance $d_{\mathrm{Xe}-\mathrm{Cu}}$. This height was calculated on $I=1 \mathrm{nA}$ and $V=10 \mathrm{mV}$ constant-current scans. The saturation for large $d$ is due to the limited size of the tip apex. The dashed line indicates the $D Z_{\max }$ variation for a sharper tip apex.

on $\mathrm{Cu}(110)$. The shape of the Xe atom is in good agreement with the shape determined experimentally. ${ }^{7}$ Moreover, due to the rectangular character of the $\mathrm{Cu}(110)$ lattice, the bump is found to be slightly asymmetric, as observed in the Eigler's experiment. ${ }^{7}$ We also find $0.17 \AA$ for the naked $\mathrm{Cu}(110)$ corrugation. This is a small apparent corrugation resulting from the fact that $\mathrm{Cu}(110)$ does not reconstruct.

\section{MOVING A Xe ATOM ON Cu(110)}

When the STM tip apex is brought close to the substrate (above or near the adsorbate), the potential energy of the adsorbate is modified relative to its value in $\mathrm{ab}$ sence of the tip. The new total energy $U\left(\mathbf{R}, \mathbf{R}_{0}\right)$ of the tip-apex-adsorbate-surface system can be written as

$$
U\left(\mathbf{R}, \mathbf{R}_{0}\right)=U_{S}+U_{D}+V_{S}+V_{D} .
$$

In this summation, potentials $U_{S}$ and $U_{D}$ are as defined previously. $\quad V_{S}$ is a short-range repulsive term due to the overlap between the electronic clouds of the adsorbate and the tip apex at very short distance. It is a pairwise summation of Born-Mayer potentials such as that used in Sec. II. $V_{D}$ comes from long-range dispersive forces. Such forces are due to correlation effects between the polarization fluctuations of the electron cloud of each atom of the tip apex and the xenon atom. Theoretical expressions of this energy are available for simple geometries (atom on a plane, atom near a sphere, etc). But little work has been devoted to the specific geometry of a metallic tip apex positioned above an atom. The coupledmodes method provides a convenient framework for studying such a geometry. It describes the dispersion energy between an atom and an STM tip apex in the following way: ${ }^{32}$ the allowed coupled modes between a $n$-atom tip apex and an adsorbate are solutions of the standard dispersion relation

$$
D(\omega)=\operatorname{det}\left[\mathbf{I}-\mathbf{B}\left(\mathbf{R}, \mathbf{R}_{0}, \omega\right)\right],
$$

where $I$ is the identity matrix and $B$ is the $(3 n \times 3 n)$ matrix defined by

$$
\mathbf{B}\left(\mathbf{R}, \mathbf{R}_{0}, \omega\right)=\alpha_{t}(\omega)\left[\begin{array}{c}
\mathbf{S}_{11}^{(a)} \mathbf{H}_{12}^{(a)} \cdots \mathbf{H}_{1 n}^{(a)} \\
\mathbf{H}_{21}^{(a)} \mathbf{S}_{22}^{(a)} \cdots \mathbf{H}_{2 n}^{(a)} \\
\vdots \\
\mathbf{H}_{n 1}^{(a)} \cdots \mathbf{S}_{n n}^{(a)}
\end{array}\right] .
$$

Here, $\alpha_{t}(\omega)$ represents the effective dipolar polarizability of an atom of the tip apex. $\mathbf{S}_{i j}^{(a)} \equiv \mathbf{S}^{(a)}\left(\mathbf{R}_{i}, \mathbf{R}_{j}, \omega\right)$ is the field susceptibility associated with the adsorbed atom. ${ }^{32}$ Finally, the $\mathbf{H}_{i j}^{(a)}$ terms are given by

$$
\begin{aligned}
\mathbf{H}^{(a)}\left(\mathbf{R}_{i}, \mathbf{R}_{j}, \omega\right)= & \mathbf{S}^{(a)}\left(\mathbf{R}_{i}, \mathbf{R}_{j}, \omega\right) \\
& +\frac{3\left(\mathbf{R}_{i}-\mathbf{R}_{j}\right)\left(\mathbf{R}_{i}-\mathbf{R}_{j}\right)-\mathbf{I}\left|\mathbf{R}_{i}-\mathbf{R}_{j}\right|^{2}}{\left|\mathbf{R}_{i}-\mathbf{R}_{j}\right|^{5}},
\end{aligned}
$$

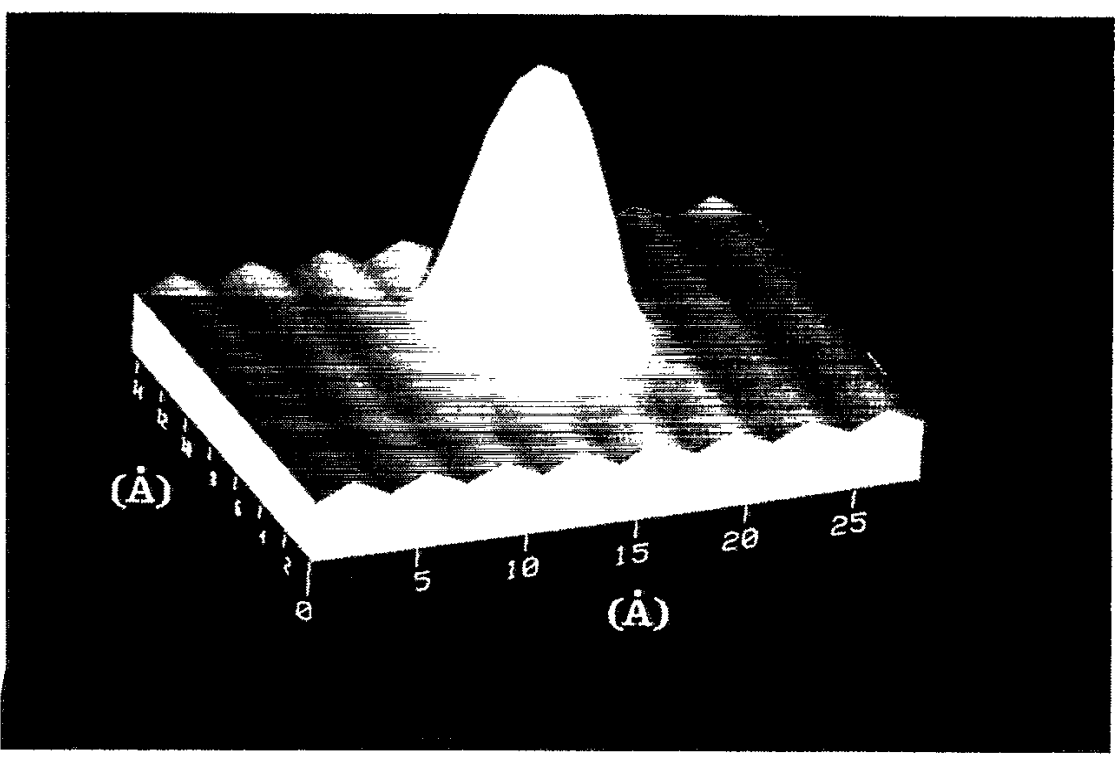

FIG. 6. Constant-current STM image of the $\mathrm{Cu}(110)$ face with a Xe atom adsorbed. $I=1$ $\mathrm{nA}, V=10 \mathrm{mV}$. The corrugation is $1.7 \AA$ from black to white in a linear gray scale. The scanned area is $28 \times 15 \AA^{2}$. 
where $\mathbf{R}_{i}=\mathbf{r}_{i}+\mathbf{R}_{0}$ is the position of the $i$ th atom of the tip.

The dispersion relation (10) is sufficient for determining the atom-tip dispersion potential energy. For interatomic distances beyond the repulsive regime, one obtains

$$
V_{D}=-\frac{\hbar}{2 \pi} \int_{0}^{+\infty} \sum_{m=1}^{\infty} \frac{1}{m} T_{r}\left[\mathbf{B}^{(m)}\left(\mathbf{R}, \mathbf{R}_{0}, i \xi\right)\right] d \xi \text {. }
$$

The tip chosen for calculating $U\left(\mathbb{R}, \mathbf{R}_{0}\right)$ is a pyramid of copper atoms as in III. It consists of four layers, each organized into a rectangular lattice respecting the order of the $\mathrm{Cu}(110)$ surface. The parameters in (12) and (13) come from Ref. 22. Using the function $U\left(\mathbf{R}, \mathbf{R}_{0}\right)$, we have plotted the potential energy $U(d)$ of a Xe atom constrained to remain on the surface while the tip apex is above its adsorption site (Figs. 7 and 8). As in Sec II, $d$ is the altitude of the $\mathrm{Xe}$ atom relative to the first atomic plane of the $\mathrm{Cu}(110)$ surface. With the tip apex kept above the hollow site, the surface binding energy $E_{b s}$ of the $\mathrm{Xe}$ atom is also plotted as a function of the lateral position of the Xe atom on the surface (Figs. 9 and 10). Three ranges of the tip-apex-to-substrate distance $Z_{0}$ can be distinguished. For large $Z_{0}\left(Z_{0}>9 \AA\right), U(d)$ is not strongly modified by the presence of the tip compared to $U(d)$ calculated without the tip (Fig. 1). For intermediate $Z_{0}\left(6 \AA<Z_{0}<9 \AA\right), U(d)$ and the $E_{\text {bs }}$ maps are strongly modified compared to the ones without the tip (Figs. 1 and 3). For small $Z_{0}\left(Z_{0}<6 \AA\right)$, the Xe energy potential is also deformed. The initial hollow site under the tip apex is no longer an equilibrium position for the $\mathrm{Xe}$ atom. Each $Z_{0}$ range with the corresponding STMinduced atom sliding process are discussed in the following.

For large $Z_{0}\left(Z_{0}>9 \AA\right), U(d)$ presents two minima for each of the Xe adsorption sites (Fig. 7). The deeper one corresponds to the adsorption of the Xe atom on the $\mathrm{Cu}(110)$ surface in the presence of the tip. On the hollow site, $E_{\mathrm{bs}}$ remains unchanged compared to the one calculated without the tip: $E_{\mathrm{bs}}=178 \mathrm{meV}$ (Fig. 7). The small-

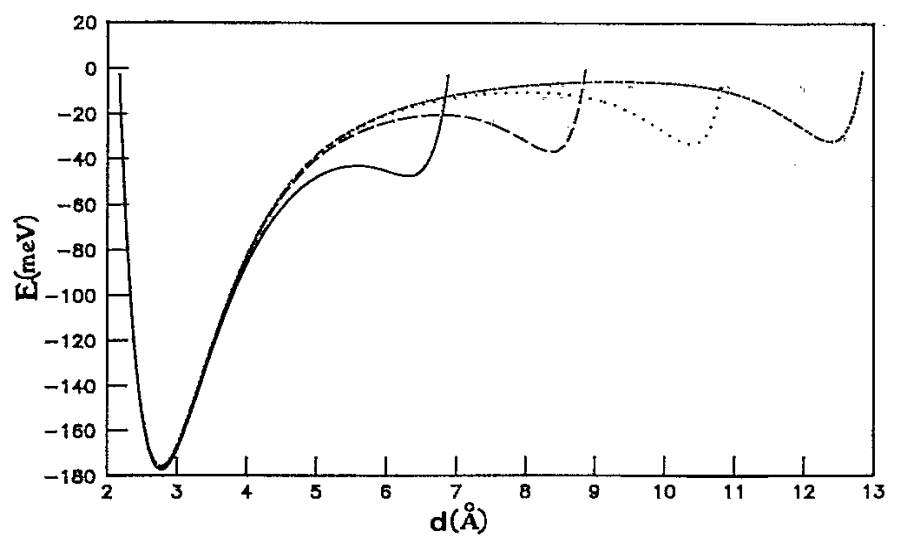

FIG. 7. Variations of the Xe potential energy $U(d)$ when the $\mathrm{Xe}$ atom is located in the gap between the tip and the surface. The tip apex and the $\mathrm{Xe}$ atom are placed above the hollow site a. ( ᄂ $Z_{0}=10 \AA$; $(----) Z_{0}=12 \AA ;(\cdots), Z_{0}=14 \AA$; and $(\cdots-\cdots-\cdots), Z_{0}=16 \AA$ with a fixed tip-apex position $\mathbf{R}_{0}=\left(a / 2, a / 2 \sqrt{2}, Z_{0}\right)$.

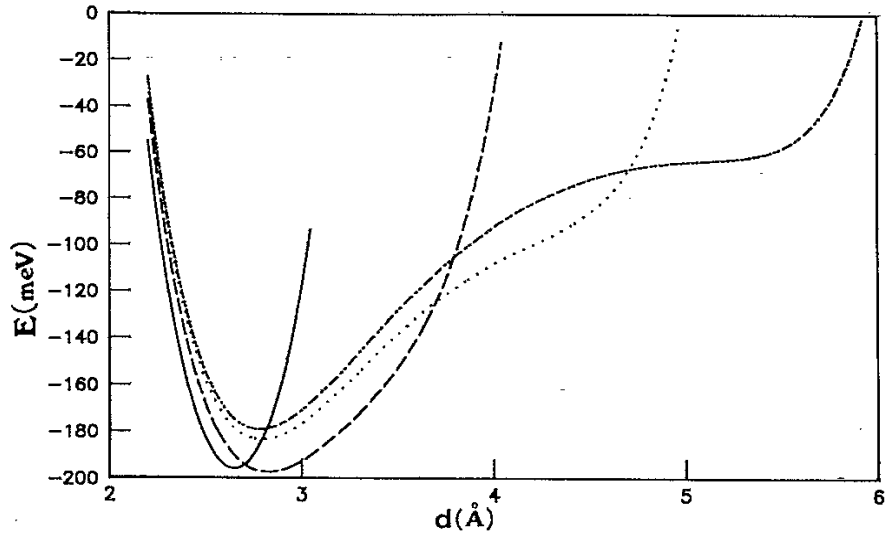

FIG. 8. Same as Fig. 7 but for shorter tip-to-substrate distances: $\left.(-), Z_{0}=6 \AA ;(---)\right) Z_{0}=7 \AA ;(\cdots), Z_{0}=8 \AA$, and $(\cdots-\cdots-\cdots), Z_{0}=9 \AA$.

er minimum corresponds to a hypothetical adsorption of the $\mathrm{Xe}$ atom on the tip-apex end atom. Above the hollow site, its binding energy on the tip, $E_{\mathrm{bt}}$, varies from 37 to $32 \mathrm{meV}$, depending on $Z_{0}$ (Fig. 7). This energy is too small to give the $\mathrm{Xe}$ atom a chance to stick on the tip apex. The respective depths of the two minima depend
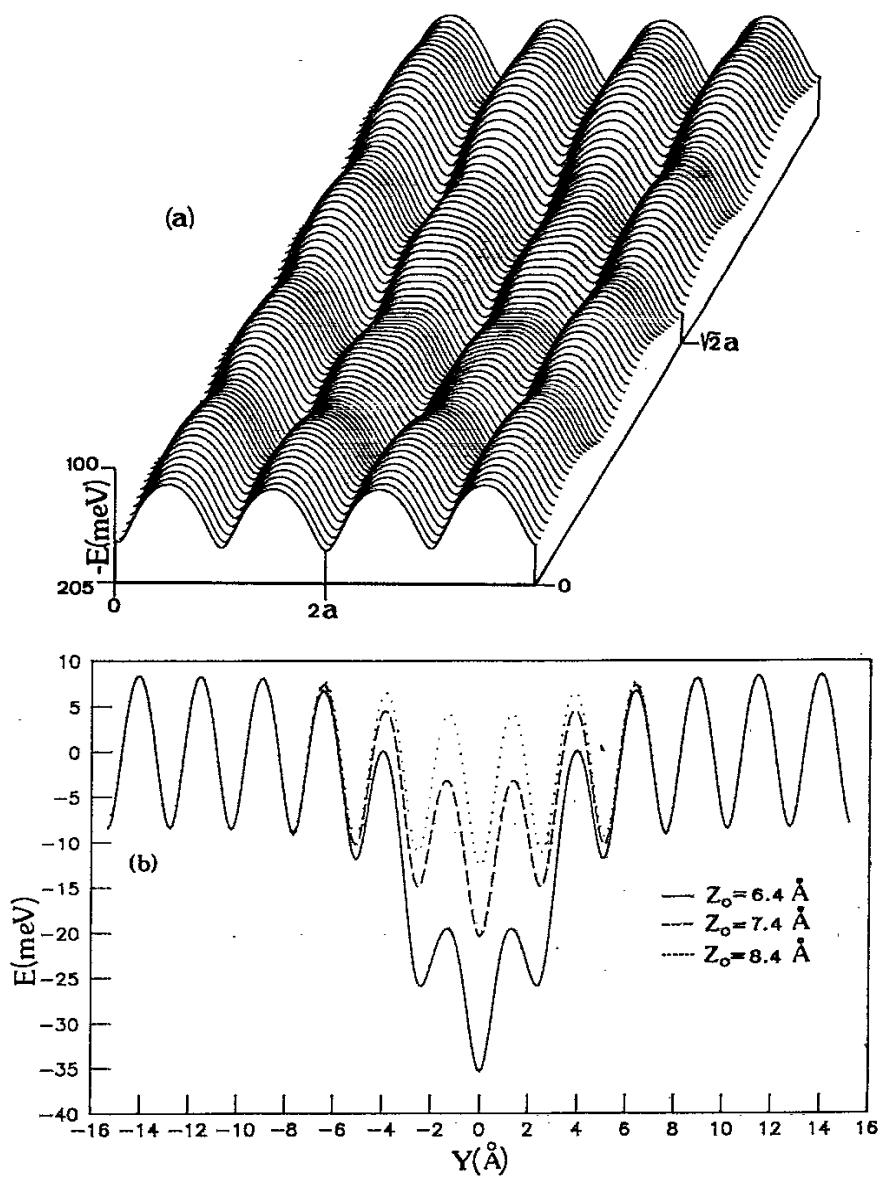

FIG. 9. Map of the minima $E_{\text {bs }}$ of the Xe potential energy $U(d)$ above the $(110) \mathrm{Cu}$ surface: The Xe scanned area is the same as in Fig. 3. (a) Map for a tip-substrate distance $Z_{0}=6.5$ $\AA$. (b) A section of this map along a bottom row for different tip altitudes. 
on the adsorption site chosen for the Xe atom.

For such large tip-to-surface distances, the $\mathrm{Cu}(110)$ surface can be scanned at constant current without the $\mathrm{Xe}$ equilibrium position being destabilized. For example, given a constant current of $10 \mathrm{pA}$ and a bias voltage of 10 $\mathrm{mV}$, the absolute $Z_{0}$ (calculated using the STM-ESQC technique described in Sec. III) is $9.74 \AA$ on the top of Xe atom and $8 \AA$ on average over the naked $\mathrm{Cu}(110)$ surface. This corresponds to a tunnel-junction resistance of $1 \mathrm{G} \Omega$, which is higher than the $5 \mathrm{M} \Omega$ threshold resistance required experimentally to slide a $\mathrm{Xe}$ atom along the $\mathrm{Ni}(\overline{1} 10)$ rows.

For intermediate $Z_{0}\left(6 \AA<Z_{0}<9 \AA\right)$ and for the tip positioned above a hollow site, there is a progressive collapse of the two previous wells into a single well. This corresponds to the formation of a tip-Xe-surface bonded object (Fig. 8). The Xe atom is constrained by the tip to stay between the tip and the surface in its hollow site. The complete collapse occurs around $Z_{0}=7 \AA$. Before then, there is still an inflection point on the $E(d)$ curves which is well marked at 9 and $8 \AA$. This is a souvenir of the tip adsorption well (Fig. 8).

More important for the understanding of the sliding process is the variation of $E_{\text {bs }}$ in the hollow site when the
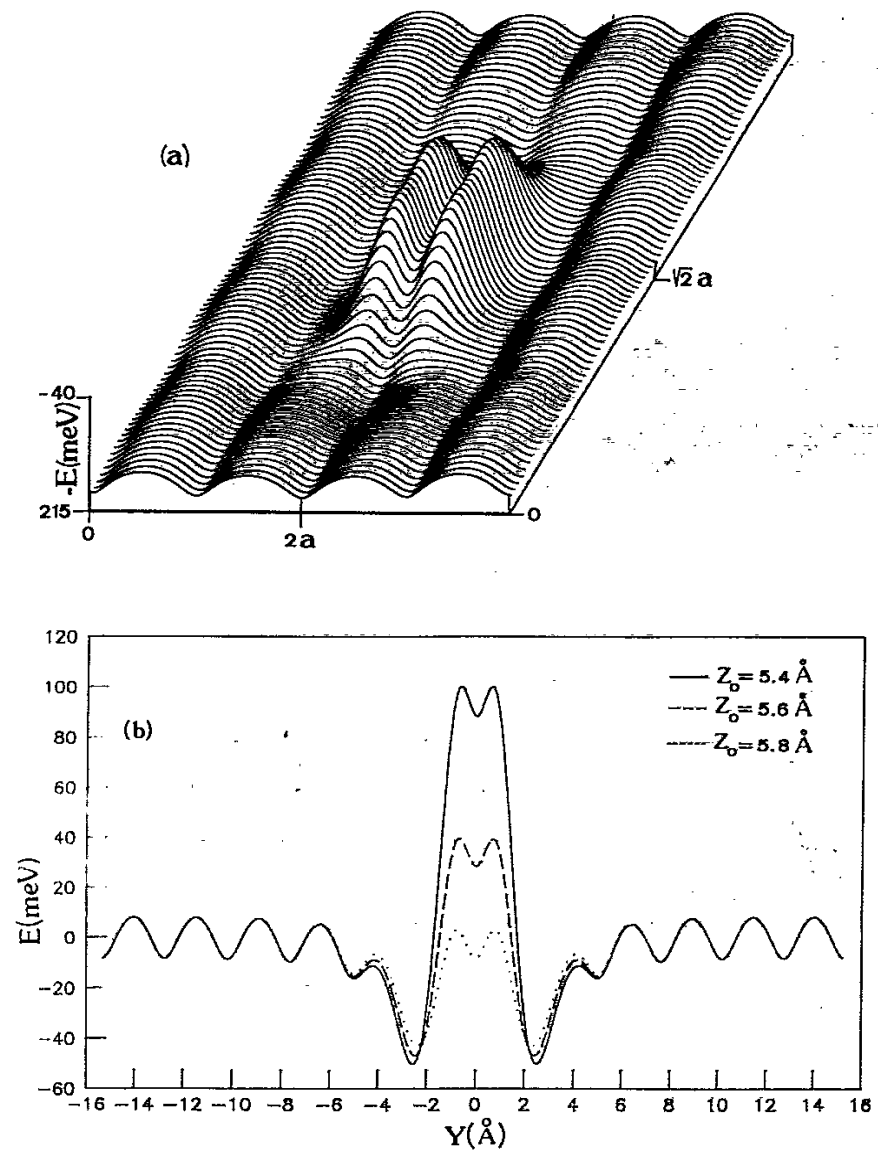

FIG. 10. Map of the minima $E_{\text {bs }}$ of the Xe potential energy $U(d)$ above the (110) Cu surface. The Xe scanned area is the same as in Fig. 3. (a) Map of a tip-substrate distance $Z_{0}=5.4$ $\AA$. (b) A section of this map along a bottom row for different tip altitudes. tip apex approaches the $\mathrm{Xe}$ atom. $E_{\mathrm{bs}}$ starts at $178 \mathrm{meV}$ for $Z_{0}=9 \AA$ and rises to $205 \mathrm{meV}$ for $Z_{0}=6.4 \AA$. This variation can be explained by the fact that the tip blocks the $\mathrm{Xe}$ atom in its hollow site and that the tip-Xe repulsive interaction is not sufficient in the intermediate $Z_{0}$ range to push the $\mathrm{Xe}$ atom away. The binding energy also increases for the other adsorption sites. Figure 9(a) represents a map of the $E_{\text {bs }}$ variation when the tip apex is fixed at $Z_{0}=6.4 \AA$ above a hollow site when the Xe atom is moved around laterally. To make the $E_{\text {bs }}$ increase clear, a cross section of this map along a ( $\overline{1} 10)$ bottom row is presented in Fig. 9(b). Let us point out the small extension of the deformation in the neighborhood of the hollow site under the tip apex. The van der Waals interaction is well localized on this site. This would not be the case for a perturbation created by a strong electric field between the tip and the surface.

For $I=1 \mathrm{nA}$ and $V=10 \mathrm{mV}$, the $Z_{0}$ calculated using the STM-ESQC method is $7.3 \AA$ when the tip apex is above a Xe hollow site. This is in the $Z_{0}$ range where one can still distinguish the tip and surface wells in $U(d)$. Therefore, a scan at $1 \mathrm{nA}$ constant current will not destabilize Xe out of its equilibrium site. In fact, the $E_{\mathrm{bs}}$ map is slightly deformed for this altitude [Fig. 9(b)]. But the well created is less than $10 \mathrm{meV}$ lower than the one observed for $E_{\text {bs }}$ on the free surface. The tip-Xe-surface tunnel-junction resistance is equal to $10 \mathrm{M} \Omega$, which is again higher than the $5-\mathrm{M} \Omega$ threshold resistance. Furthermore, if the setup current is increased so as to reduce the junction resistance under. $5 \mathrm{M} \Omega$, the calculated $Z_{0}$ is under $6.4 \AA$. For example, a $1-\mathrm{M} \Omega$ resistance (or $I=10$ $\mathrm{nA}, V=10 \mathrm{mV}$ ) leads to $Z_{0}=5.8 \AA$ ). Therefore, for a current intensity higher than $5 \mathrm{nA}$, the $\mathrm{Xe}$ atom is more stable under the tip apex, in the hollow site than in the other sites in the same row. If, under these conditions, the tip is moved along the row, the Xe atom trapped under the tip also moves. This analysis supposes that the deformation of the potential-energy surface follows the tip-apex displacement adiabatically and that the Xe atom always relaxes into the hollow site with the largest $E_{\text {bs }}$.

For small $Z_{0}\left(Z_{0}<6 \AA\right), E_{\text {bs }}$ is lower, since the repulsive part of the tip-Xe interaction becomes larger than the attractive part. The $E_{\text {bs }}$ maps indicate that the potential energy at the hollow site above which the tip is positioned is much higher than the potential energy at the other hollow sites where the tip is not present (Fig. 10). Therefore, $\mathrm{Xe}$ must leave its initial size and move toward another hollow site in the same row or in another row. Such an escape channel appears for $Z_{0}<5.4 \AA$, i.e., for a theoretical tunnel-current intensity higher than $40 \mathrm{nA}$. Consequently, if this intensity is increased, the tip apex is brought closer to the substrate and the Xe atom is destabilized. In this case, the $\mathrm{Xe}$ atom cannot be stabilized on the nearby hollow site due to the kinetic energy gain during its collision with the tip apex. Therefore, the sliding can only be explained by the van der Waals process detailed for the intermediate $Z_{0}$ distance range.

\section{v. CONCLUSION}

When the STM tip scans at a low tunneling current over a $\mathrm{Cu}(110)$ surface upon which a $\mathrm{Xe}$ atom has been 
adsorbed, the Xe atom keeps its hollow-site equilibrium position. At its $2.78-\AA$ equilibrium distance above the surface, it produces a bump $1.6 \AA$ high in the STM image $(I=1 \mathrm{nA}, V=10 \mathrm{mV})$. If the tip-to-surface distance is reduced in such a way that the Xe atom is constrained in its initial hollow site between the tip apex and the surface, this atom can slide on the surface following the attractive depression created by the tip apex on the hollow site. We have determined theoretically that this sliding process occurs for a tunneling-current range (or a tunnel-junction resistance range) which is in good agreement with the experimental range found by Eigler and co-workers. For a given adsorbate, the sliding process appears limited to a precise tunnel-resistance range. For xenon on copper, this is between 1 and $5 \mathrm{M} \Omega$. If the current increases further, the collision between the tip apex and the Xe atom will force the Xe atom out of its initial equilibrium site.

A comparison between the experimental data and our results shows that all our absolute tip-apex-to-substrate distances appear to be underestimated by $0.3-0.6 \AA$.
However, it is well recognized ${ }^{13}$ that the experimental threshold resistance necessary to slide an adsorbate depends on the particular arrangement of the tip-apex atom by a factor estimated to be less than 4 . A factor of 4 in the junction resistance leads to a precision in the altitude not better than $0.5 \AA$. Moreover, the tip apex used in our calculation is made of copper and not of tungsten, with a tetragonal instead of a trigonal base. This is not the tip used by Eigler and co-workers. This may explain the small discrepancies observed. Note also that our calculations suppose that the substrate and the tip apex are rigid. Therefore, the deep well in the $E_{\text {bs }}$ map [Fig. 9(b)] is perhaps less pronounced in the experiment than in the calculated map.

\section{ACKNOWLEDGMENTS}

We would like to thank the CNRS program Ultimatech for financial support and Fujitsu France for computing support during this work.
${ }^{1}$ M. A. Chesters, M. Hussain, and J. Pritchard, Surf. Sci. 35, 161 (1973).

${ }^{2} \mathrm{M}$. Bienfait, Surf. Sci. 89, 13 (1979), and references therein.

${ }^{3}$ J. Unguris, L. W. Bruch, E. R. Moog, and M. B. Webb, Surf. Sci. 109, 522 (1981).

${ }^{4}$ A. Glachant and U. Bardi, Surf. Sci. 87, 187 (1979); A. Glachant, Ph.D. thesis, Aix-Marseille University, 1981; K. Christmann and J. Demuth, Surf. Sci. 120, 291 (1982).

${ }^{{ }^{5}}$ H. Ohtani, R. J. Wilson, S. Chiang, and C. M. Mate, Phys. Rev. Lett. 60, 2398 (1988); P. H. Lippel, R. J. Wilson, M. D. Miller, C. H. Wöll, and S. Chiang, ibid. 62, 171 (1989).

${ }^{6} J$. K. Gimzewski, E. Stoll, and R. R. Schlittler, Surf. Sci. 181, 267 (1987).

${ }^{7}$ D. M. Eigler and E. K. Schweizer, Nature 344, 524 (1990).

${ }^{8}$ D. M. Eigler, P. S. Weiss, E. K. Schweizer, and N. D. Lang, Phys. Rev. Lett. 66, 1189 (1991).

${ }^{9}$ D. M. Eigler, C. P. Lutz, and W. E. Rudge, Nature 352, 600 (1991).

${ }^{10}$ I. W. Lyo and Ph. Avouris, J. Chem. Phys. 93, 4479 (1990).

11 J. S. Foster, J. E. Frommer, and P. C. Arnett, Nature 331, 325 (1988).

${ }^{12}$ L. J. Whitman, J. A. Stroscio, R. A. Dragoset, and R. J. Celotta, Science 251, 1206 (1991).

13J. A. Stroscio and D. M. Eigler, Science 254, 1319 (1991).

${ }^{14}$ G. Vidali, G. Ihm, H. Y. Kim, and M. W. Cole, Surf. Sci. Rep. 12, 133 (1991).

${ }^{15}$ N. R. Hill, M. Haller, and V. Celli, Chem. Phys. 73, 363
(1982); V. Celli, D. Eichenauer, A. Kaufhold, and J. P. Toennies, J. Chem. Phys. 83, 2504 (1985).

${ }^{16}$ G. Vidali, M. W. Cole, and J. K. Klein, Phys. Rev. B 28, 3064 (1983).

${ }^{17}$ A. D. Stone and A. Szafer, IBM J. Res. Dev. 32, 384 (1988).

${ }^{18}$ R. Hoffmann, J. Chem. Phys. 39, 1397 (1963).

${ }^{19}$ C. Girard and C. Girardet, Phys. Rev. B 36, 909 (1987).

${ }^{20}$ C. Girard, J. Chem. Phys. 85, 6750 (1986).

${ }^{21}$ X. P. Jiang, F. Toigo, and M. W. Cole, Surf. Sci. 145, 281 (1984).

${ }^{22}$ C. Girard and J. Humbert, Chem. Phys. 97, 87 (1985).

${ }^{23}$ P. Sautet and C. Joachim, Chem. Phys. Lett. 153, 511 (1988); 165, 99 (1989).

${ }^{24}$ P. Sautet and C. Joachim, Chem. Phys. Lett. 185, 23 (1991).

${ }^{25}$ P. Sautet and C. Joachim, Surf. Sci. 271, 387 (1992).

${ }^{26}$ P. Sautet and C. Joachim, Ultramicroscopy 42, 115 (1992).

${ }^{27}$ J. Dunphy, D. F. Ogletree, M. B. Salmeron, P. Sautet, and M. L. Bocquet, and C. Joachim, Ultramicroscopy 42, 490 (1992).

${ }^{28}$ A. Farazdel and M. Dupuis, Phys. Rev. B 44, 3909 (1991).

${ }^{29}$ P. W. Anderson, D. J. Thouless, E. A. Abrahams, and D. S. Fisher, Phys. Rev. B 22, 3519 (1980).

${ }^{30} \mathrm{C}$. Joachim and P. Sautet, in Scanning Tunneling Microscopy and Related Methods, edited by R. J. Behm, N. Garcia, and H. Röhrer (Kluwer, Dordrecht, 1990), p. 377.

${ }^{31}$ J. Bardeen, Phys. Rev. Lett. 6, 57 (1961).

${ }^{32}$ C. Girard and X. Bouju, J. Chem. Phys. 95, 2056 (1991). 